\title{
Uterine endometrial gene expression in heifers of high or low fertility on day 14 of the estrous
} cycle

\author{
AP Killeen ${ }^{1,2}$, DG Morris ${ }^{3}$, DA Kenny ${ }^{2}$, MG Diskin $^{3}$, SM Waters ${ }^{1}$
}

${ }^{1}$ Teagasc Animal Bioscience Centre, Grange, Meath, Ireland, ${ }^{2}$ School of Agriculture, Food Science \& Veterinary Medicine, University College Dublin, Bellfield, Dublin 4, Ireland, ${ }^{3}$ Teagasc Animal Bioscience Centre, Mellows Campus, Athenry, Galway, Ireland

Email: aideen.killeen@teagasc.ie

Introduction Early embryo loss, occurring within the first two weeks of pregnancy, constitutes $70-80 \%$ of reproductive wastage (Diskin et al., 2002). Repeatable differences between heifers in their ability to become pregnant have been established and uterine rather than ovarian factors are generally thought to account for these differences (McMillan et al., 2001). However, the biochemical mechanisms governing uterine mediated early embryo development, or indeed the specific timeframe within the first two weeks of pregnancy during which most loss occurs, are not yet known. We previously reported that a number of genes with identified roles in embryonic development were differentially expressed in uterine endometrial tissue on day 7 of the estrous cycle, between heifers ranked either high or low for fertility (Killeen et $a l ., 2009)$. The aim of the current study was to further elucidate the underlying molecular mechanisms contributing to early embryo loss by examining differential uterine endometrial gene expression of high or low fertility heifers on day 14 of the estrous cycle.

Material and methods Reproductively normal crossbred beef heifers $(n=120)$ were inseminated and pregnancy diagnosis was carried out 28 days later, after which animals were programmed to return to estrus. Animals were re-inseminated followed by pregnancy diagnosis on a further four occasions. On the basis of pregnancy rate to 5 successive inseminations, animals were divided into two groups: i) those that established pregnancy on all 5 occasions (high fertility group) and ii) animals achieving pregnancy on only one occasion (low fertility group). Estrous cycles were then synchronised following administration of a prostaglandin $\mathrm{F} 2_{\alpha}$ analogue (Estrumate $\left.{ }^{\circledR}\right)$. Animals from both high $(\mathrm{n}=7)$ and low groups (n=7) were slaughtered on day 14 of the estrous cycle and uterine endometrial tissue (ipsilateral to corpus luteum) was harvested and stored at $-80^{\circ} \mathrm{C}$ in RNAlater solution. RNA was extracted using TRIzol® reagent, purified, DNAse treated, and quantified and quality control checked using the 2100 Agilent Bioanalyser. RNA samples were then biotinylated and hybridised to the Affymetrix 23,000 bovine master Gene Chip according to manufacturers' protocol. Normalisation and statistical analysis were carried out using R and the PUMA method in Bioconductor (Pearson et al., 2009). Functional analyses were generated through the use of Ingenuity Pathway Analysis (IPA, CA, USA).

Results Differentially expressed genes (DEGs) totalled $430(P<0.05), 156$ of these were up-regulated and 274 downregulated. Genes mapped were implicated in a host of biological pathways including lipid metabolism, LDLR, PTGS2, PCCB, ACOT4 \& ALOX5; cellular growth and proliferation, PPARG, ABTB1, HCRT, BRAF \& MC4R; vitamin and mineral metabolism, SLC27A4, G6PC, ARG2 \& CYP4F3; nutritional diseases, MC4R \& LDLR; inflammatory response, APRT, PTGS2 \& C6 and embryonic development, PPARG, BRAF \& ITGA6. The top canonical pathways generated found to contribute to low conception rate, included mitochondrial function, citrate cycle, oxidative phosphorylation and linoleic acid metabolism. The many mapped canonical pathways, while simultaneously contributing to an extensive list of generated biological processes (Figure 1), illustrates the likely multifactorial nature of endometrial function between high and low fertility animals. In agreement with these findings, a previous study by our group (Killeen et al., 2009) using endometrial tissue collected on day 7 of the estrous cycle show similar transcript profiles between high and low fertility heifers. For example, DEGs expressed similarly in both studies include: PCCB, SLC25A24, DAP and COL4A4 $(P<0.05)$. Of interest, PCCB; the gene encoding propionyl-CoA carboxylase beta polypeptide, is contained within the blepharophimosis-ptosis-epicanthus inversus syndrome (BPES) locus. There are two types of this congenital defect: BPES I \& II. Female infertility differentiates the former from the latter (Piemontese et al., 1997). Thus, future work will determine if variants of this gene could potentially serve as a marker for fertility in cattle.

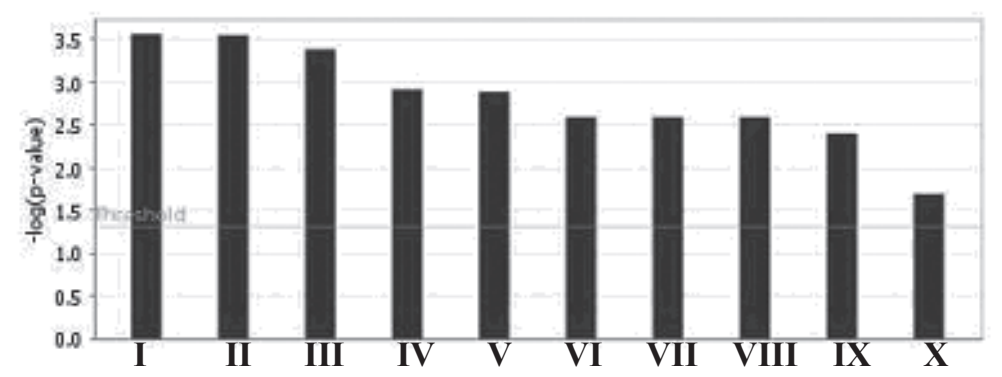

Figure 1 Key gene networks expressed in bovine endometrial tissue.

$\begin{array}{ll}\text { I } & \text { Organismal Functions } \\ \text { II } & \text { Lipid Metabolism } \\ \text { III } & \text { Vitamin \& Mineral Metabolism } \\ \text { IV } & \text { Cell Growth \& Proliferation } \\ \text { V } & \text { Nutritional Disease } \\ \text { VI } & \text { Cell Morphology } \\ \text { VII } & \text { Haematological System } \\ \text { VIII } & \text { Inflammatory Response } \\ \text { IX } & \text { Tissue Morphology } \\ \text { X } & \text { Embryonic Development }\end{array}$

Conclusion Expression of genes involved in key biological pathways including embryonic development are differentially regulated in the uterine endometrium of heifers of high compared with low fertility on day 14 of the estrous cycle. Acknowledgements The authors greatly acknowledge funding from Teagasc.

References Diskin MG, Kenny DA, Dunne L and Sreenan JM. 2002. Proceeding of the Irish Agric Research Forum.,27 McMillan WH, Peterson AJ, Cox SF, Person SJ and Donnison MJ. 2001. British Society of Animal Production, $283-288$. Killeen AP, Morris, DG, Kenny DA, Diskin MG and Waters SM. 2009. Proceedings of the Irish Agric Res Forum, 150. Pearson R, Liu X, Sanguinetti G, Milo M, Lawrence ND and Rattray M. 2009. BMC Bioinformatics 10, 211. Piemontese MR, Memeo E, Carella M, Amati P, Chomel JC, Bonneau D, Pilia G, Cao A, Drabkin H, Gemmill R, Rommens J, Zelante L, Gasparini P and Bisceglia L. 1997. European Journal of Human Genetics 5(3), 171-174. 